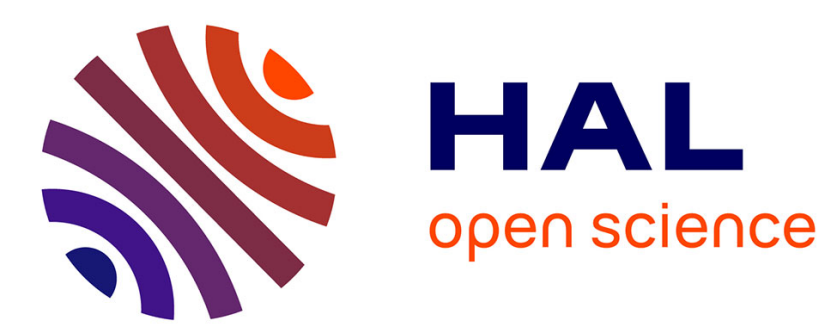

\title{
Un laser à verre dopé au néodyme fortement cohérent
}

\author{
C. Aplin, J. Fleuret, N. Gaggioli
}

\section{To cite this version:}

C. Aplin, J. Fleuret, N. Gaggioli. Un laser à verre dopé au néodyme fortement cohérent. Revue de Physique Appliquée, 1970, 5 (6), pp.841-844. 10.1051/rphysap:0197000506084100 . jpa-00243463

\section{HAL Id: jpa-00243463 https://hal.science/jpa-00243463}

Submitted on 1 Jan 1970

HAL is a multi-disciplinary open access archive for the deposit and dissemination of scientific research documents, whether they are published or not. The documents may come from teaching and research institutions in France or abroad, or from public or private research centers.
L'archive ouverte pluridisciplinaire HAL, est destinée au dépôt et à la diffusion de documents scientifiques de niveau recherche, publiés ou non, émanant des établissements d'enseignement et de recherche français ou étrangers, des laboratoires publics ou privés. 


\title{
UN LASER A VERRE DOPÉ AU NÉODYME FORTEMENT COHÉRENT
}

\author{
C. APLin, J. FleUret (**) et N. GAGGiOli $(* * *)$
}

Laboratoires de Marcoussis, Compagnie Générale d'Electricité

(Reçu le 10 juillet 1970)

\begin{abstract}
Résumé. - Nous avons obtenu un laser à verre dopé au néodyme fortement cohérent en diminuant le nombre de Fresnel de la cavité oscillatrice, de façon à filtrer le mode fondamental.

La divergence est alors limitée par la diffraction à $5 \times 10^{-5} \mathrm{rd}$, pour une surface émettrice de $23 \mathrm{~mm}$ de diamètre ; par conséquent, la luminance atteint sa valeur maximum $\left(1,7 \times 10^{16} \mathrm{~W} / \mathrm{St}\right.$. $\mathrm{cm}^{2}$ ).

Nous avons obtenu à la sortie, dans l'infrarouge, l'énergie totale de 10 joules, avec une très bonne cohérence spatio-temporelle. Nous décrivons ici la géométrie du laser, ainsi que les méthodes utilisées pour améliorer la source et pour mesurer la cohérence.

Abstract. - A highly coherent Nd-glass laser has been achieved by decreasing the Fresnel number of a Nd-glass laser cavity so as to obtain the fundamental mode. Beamspread is diffraction-limited $\left(5 \times 10^{-5} \mathrm{rd}\right.$ for an emissive area $23 \mathrm{~mm}$ in diameter and, consequently, radiance is maximized $\left(1.7 \times 10^{16} \mathrm{~W} / \mathrm{St} . \mathrm{cm}^{2}\right)$.

A total infrared $10 \mathrm{~J}$ output is obtained with high spatial and temporal coherence; the geometry of the laser and the methods used to improve source and measure coherence are described.
\end{abstract}

Introduction. - Il est souhaitable, pour les applications en holographie ultra-rapide, de posséder un laser déclenché délivrant une énergie élevée, et fortement cohérent. Plusieurs auteurs [1 à 5] ont déjà étudié des lasers à rubis déclenchés monomodes. W. F. Hagen [6] a récemment étudié un laser à verre dopé au néodyme de forte luminance.

Dans cet article, nous examinons avec quelques détails un oscillateur laser à verre dopé au néodyme, déclenché par cellule de Kerr, et dans la cavité duquel nous avons placé un diaphragme. On montre que cette cavité délivre une radiation monomode de très bonne qualité quant à la cohérence, la divergence et la luminance. Après amplification, la luminance augmente alors que la cohérence est très peu modifiée ; la divergence reste limitée par la diffraction.

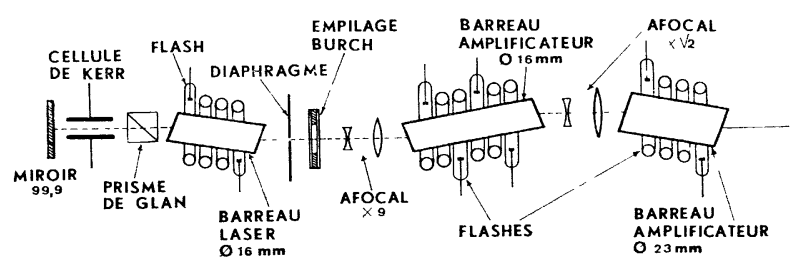

FIG. 1. - Schéma du dispositif expérimental.

$\left(^{*}\right)$ Cette étude a bénéficié du soutien de la Direction des Recherches et Moyens d'Essais, division Optique.

$(* *)$ adresse actuelle :

Ecole Nationale Supérieure des Télécommunications,

46, rue Barrault. Paris $13^{\mathrm{e}}$.

$(* * *)$ Chercheur de l'Institut de Recherches Scientifiques et Techniques des Forces Armées d'Argentine. Adresse actuelle: Institut d'Optique, 3 bd Pasteur, Paris $15^{\mathrm{e}}$.
Dispositif expérimental. - Le dispositif expérimental est schématisé figure 1. L'oscillateur comporte un barreau de verre dopé au néodyme (dopage $5 \%$ ) de $260 \mathrm{~mm}$ de long et $16 \mathrm{~mm}$ de diamètre, à extrémités taillées en biseau de $6^{\circ}$. Le barreau (bords exceptés) est plongé dans une solution de chromate de potassium à $2 \mathrm{~g} / \mathrm{l}$. Un flash hélicoïdal délivre une impulsion de $0,8 \mathrm{~ms}$.

L'oscillateur est déclenché par une cellule de Kerr que commande une impulsion de $8 \mathrm{kV}$ et $15 \mathrm{~ns}$ de temps de montée. La distance inter-électrode est $8 \mathrm{~mm}$. Un prisme de Glan polarise le faisceau verticalement. Le résonateur est formé, à une extrémité, par un miroir plan (coefficient de réflexion $99,9 \%$ ) et à l'autre, par un filtre interférentiel du type Burch.

A la suite de l'oscillateur, nous avons placé deux amplificateurs, afin d'augmenter l'énergie cohérente. Ces deux amplificateurs sont formés de barreaux de verre dopé au néodyme de longueur $500 \mathrm{~mm}$ et diamètre $16 \mathrm{~mm}$ pour le premier, $330 \mathrm{~mm}$ et $23 \mathrm{~mm}$ respectivement pour le second. Le premier amplificateur est couplé à l'oscillateur par une lunette afocale de rapport 9. Un autre afocal de rapport $\sqrt{2}$ est placé avant le second amplificateur. Les deux afocaux sont des systèmes à lentilles prismatiques, afin d'éviter les réflexions en retour. Nous avons placé le premier afocal juste avant le premier maximum d'énergie sur l'axe, à environ $60 \mathrm{~cm}$ du diaphragme.

Nous avons étudié, à la sortie de l'oscillateur, et après amplification, l'impulsion, la cohérence spatiale et temporelle, l'énergie, la divergence et la luminance.

Sélection de modes. - Sélection des Modes Transversaux. - La sélection des modes transversaux a été 
obtenue en introduisant des pertes additionnelles pour les modes d'ordre supérieur. La méthode a déjà reçu quelques justifications théoriques [8,9].

Nous avons disposé un diaphragme à l'intérieur de la cavité. Ce diaphragme a été percé dans une feuille de clinquant de $0,4 \mathrm{~mm}$ d'épaisseur, et centré sur l'axe optique. Plusieurs diamètres ont été essayés, de 0,6 à $2,3 \mathrm{~mm}$ par pas de $0,1 \mathrm{~mm}$. Nous avons adopté la valeur définitive de $1,6 \mathrm{~mm}$ en faisant un compromis entre le caractère monomode de l'émission, et la stabilité du système. Pour chaque diamètre, nous avons mesuré le seuil de l'effet laser. Nous avons également défini un seuil «monomode » par l'énergie de pompage au-dessus de laquelle l'oscillation n'est plus monomode transverse. Entre ces deux seuils, on obtient une oscillation monomode, d'après les constatations suivantes :

- Nous avons étudié la distribution d'énergie en fonction de la distance (distance mesurée à partir du diaphragme). Nous avons trouvé une succession d'an-

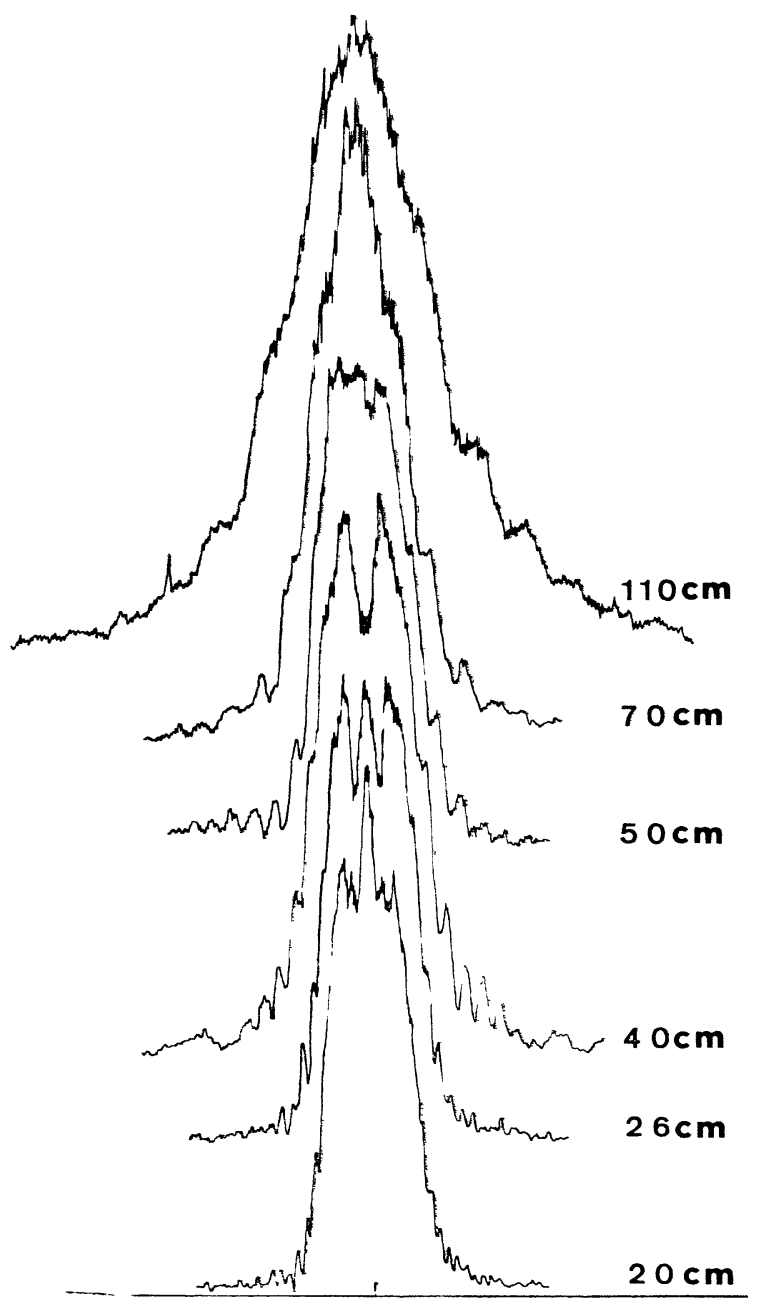

neaux de Fresnel, avec des points centraux brillants ou sombres suivant la distance. Avec l'éloignement, ces anneaux se fondent en une répartition d'énergie gaussienne. La figure 2 montre les courbes de densité obtenues sur plaque Kodak 1Z. Dans la zone proche, nous avons vérifié que les extréma d'énergie sur l'axe sont éloignés du diaphragme de

$$
\frac{1}{n} \frac{a^{2}}{\lambda}=\frac{1}{n} \frac{(0,08)^{2}}{1,06} 10^{4}=\frac{60}{n} \mathrm{~cm}
$$

où $n$ est un entier positif, impair pour un maximum et pair pour un minimum.

Dans la zone lointaine, la distribution gaussienne d'intensité

$$
I=I_{0} \exp \left(-\frac{r^{2}}{r_{0}^{2}}\right)
$$

fournit, sur la plaque photographique, une répartition de densité parabolique, car

$$
D=\gamma \log _{10}\left(\frac{I}{a}\right)
$$

entraîne :

$$
D=D_{m}-\frac{\gamma}{2,3} \frac{r^{2}}{r_{0}^{2}} .
$$

- Nous avons enregistré des impacts de sortie sur film Polaroïd infrarouge $n^{0} 413$, et sur plaque AgfaGevaert 52 A 86, ainsi que sur plaque Kodak $1 Z$.

Ces impacts sont parfaitement symétriques, ce qui

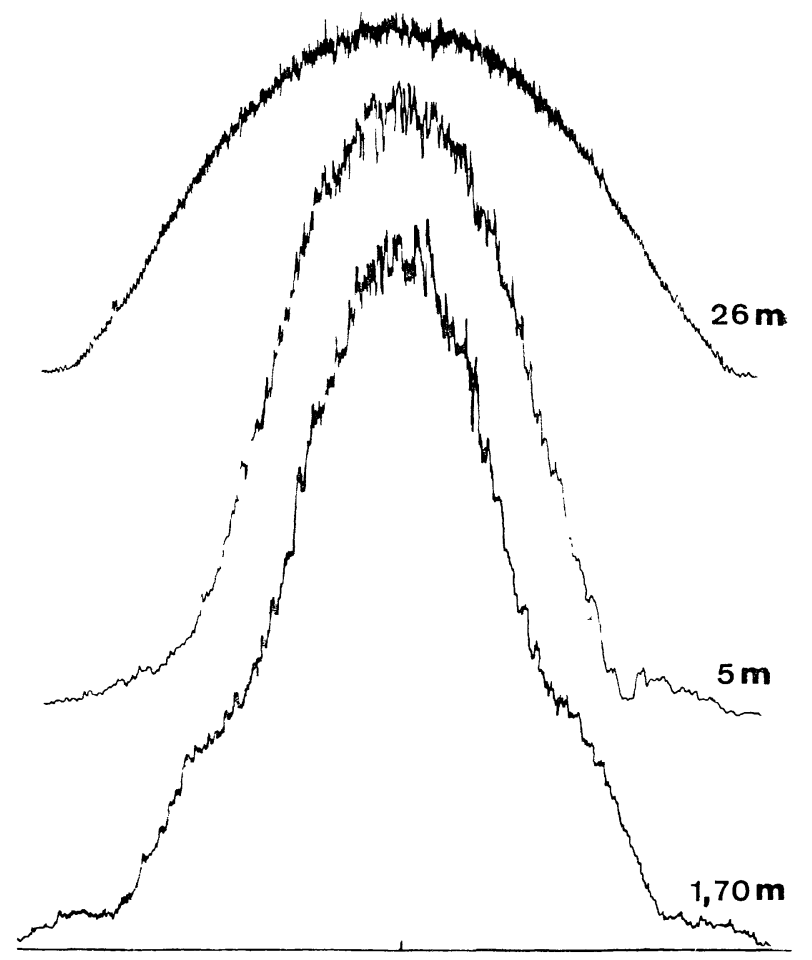

FIG. 2. - Distributions d'énergie en fonction de la distance.

Ces figures représentent la répartition d'énergie des impacts exprimée en densité sur plaque Kodak 1Z, en fonction de la distance mesurée à partir du diaphragme.

2a. - Evolution des anneaux de Fresnel jusqu’à $1,10 \mathrm{~m}$ (les courbes sont à la même échelle). 2b. - Evolution qualitative des impacts dans la zone lointaine. 
caractérise bien le mode fondamental. Au-dessus du seuil monomode, on obtient des formes d'impact caractérisant les modes d'ordre supérieur, avec au moins deux points brillants ou sombres.

Tous ces résultats concordent parfaitement avec le schéma d'une onde plane gaussienne diffractée par un diaphragme circulaire.

SÉlection Des Modes Longitudinaux. - Nous avons également observé une sélection des modes longitudinaux.

Nous avons étudié l'impulsion au moyen d'une cellule rapide suivie d'un oscilloscope Tektronix 519. Nous avons trouvé une largeur de 40 ns à mi-hauteur. La figure 3 montre la forme d'impulsion observée.
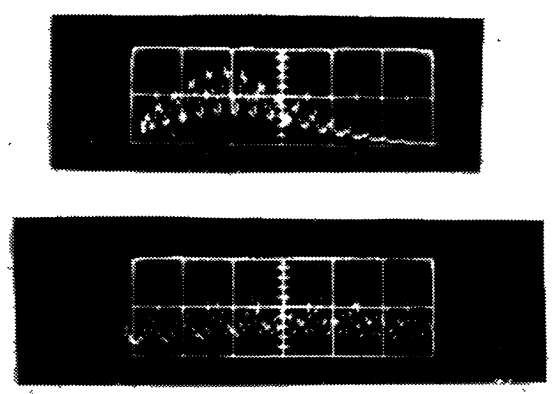

Frg. 3. - Impulsions obtenues à la sortie de l'oscillateur. En haut $20 \mathrm{~ns} / \mathrm{div}$ En bas $10 \mathrm{~ns} / \mathrm{div}$.

Comme dans la référence 1 , l'impulsion contient des oscillations additionnelles, qui semblent correspondre aux fréquences de battement entre modes axiaux. L'écart de fréquence entre deux modes axiaux consécutifs est approximativement :

$$
\frac{c}{2 l}=150 \mathrm{MHz} \text {. }
$$

Nous avons mesuré les périodes $T=7 \mathrm{~ns}$ et $T^{\prime}=1 \mathrm{~ns}$ (voir Fig. 3). 7 ns correspond à la fréquence $143 \mathrm{MHz}$. On notera que le filtre interférentiel utilisé a une largeur à-mi hauteur

$$
\Delta \lambda=2,5 \AA .
$$

La cohérence temporelle est bien meilleure que celle que laisserait prévoir (4) (voir Cohérence).

Cohérence. - Cohérence Spatiale. - Nous avons testé la cohérence de la même façon que dans la référence [7]. Par cette méthode, on mesure la cohérence globalement. Le montage est décrit figure 4 . On voit que l'un des deux faisceaux subit trois réflexions additionnelles; la plaque photographique enregistre les interférences entre deux images dont l'une est symétrique de l'autre par rapport à un axe vertical. On obtient des franges verticales, espacées périodiquement de

$$
d=\frac{\lambda}{2 \sin (\theta / 2)}
$$
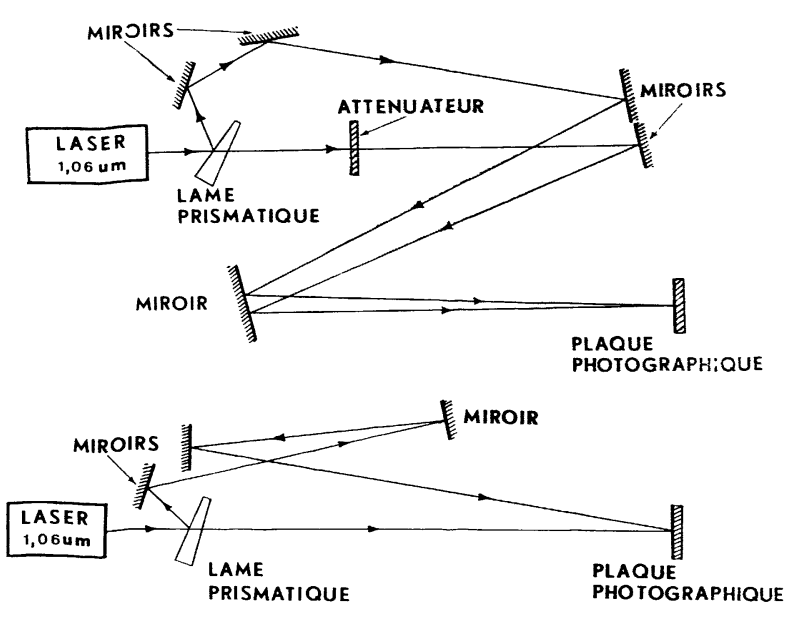

Fig. 4. - Mesure de la cohérence.

4a. - Schéma du montage utilisé pour la mesure de la cohérence spatiale (un chemin optique est $15 \mathrm{~cm}$ plus long que l'autre).

4b. - Cohérence temporelle.

où $\theta$ est l'angle des deux faisceaux.

Pour une tension de pompage de $10,5 \mathrm{kV}$, nous avons mesuré la visibilité $(*) v_{s}=0,73$ (Fig. 5). La visibilité obtenue est donc très bonne et uniforme dans tout le champ. Nous avons mesuré les visibilités pour différentes tensions de pompage. D'autres axes de symétrie ont été étudiés, fournissant également des visibilités importantes et uniformes.

Après amplification, la cohérence spatiale a été mesurée à nouveau à l'aide du même dispositif (Fig. 4). Nous avons obtenu des franges uniformes, de visibilité moyenne $v_{s}=0,42$.

Cohérence Temporelle. - Pour la mesure de la cohérence temporelle, nous avons imposé une différence paire entre les nombres de réflexions sur chaque trajet ; et l'un des trajets a été retardé dans l'air (Fig. 4). Pour la différence de marche $\delta=1 \mathrm{~m}$ nous avons obtenu la visibilité $v_{T}=0,39$.

Pour $\delta=3,4 \mathrm{~m}$ nous avons pu enregistrer des franges de $v_{T}=0,1$.

On voit que l'oscillateur a aussi une bonne cohérence temporelle. C'est ce que laissait prévoir la fréquence de

(*) La plaque photographique permet de mesurer au microdensitomètre le contraste des franges, défini à partir de densités maximum et minimum par :

$$
C=\frac{D_{M}-D_{m}}{D_{M}+D_{m}^{-}}
$$

Seule, la visibilité a une signification physique, reliée aux intensités lumineuses :

$$
v=\frac{I_{M}-I_{m}}{I_{M}+I_{m}}
$$

$C$ et $v$ sont liés par la réponse du détecteur (1) :

$$
v=\frac{10^{(C / \gamma)\left(D_{M}+D_{m}\right)}-1}{10^{(C / \gamma)\left(D_{M}+D_{m}\right)}+1}
$$



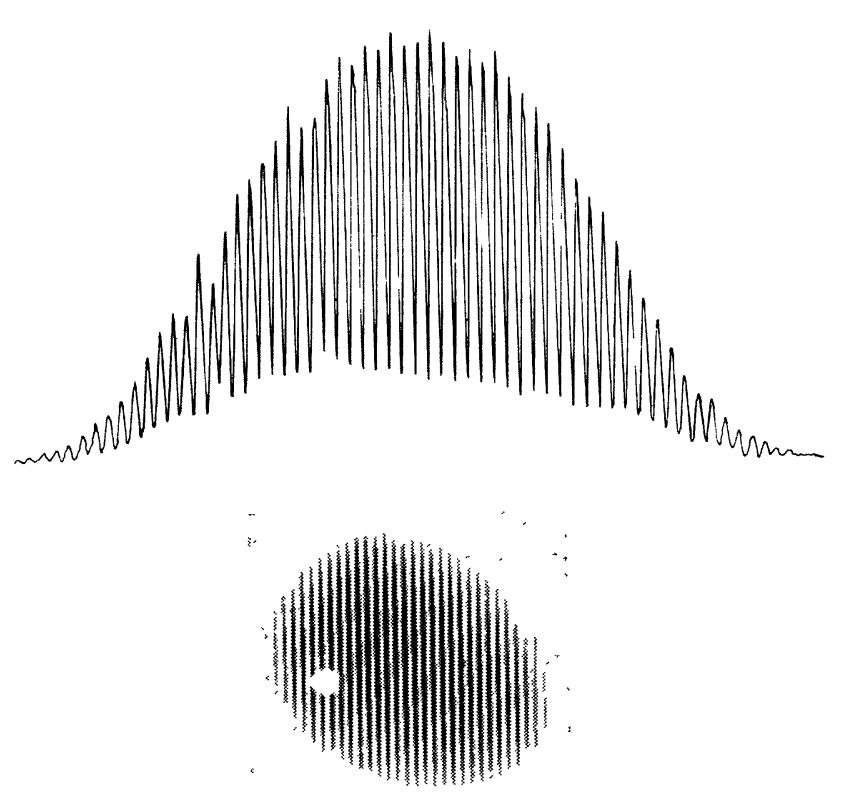

FIG. 5. - En haut :

Densitométrie des franges d'interférence obtenues lors de la mesure de la cohérence spatiale.

En bas :

Photographie des franges sur plaque $1 \mathrm{Z}$.

battement mesurée lors de l'étude de l'impulsion. D'après la référence [7], pour deux modes axiaux, la cohérence temporelle est une propriété périodique en fonction de la distance. La forme des impulsions semble montrer que nous n'avions qu'un mode axial prépondérant. Cela explique pourquoi nous avons obtenu une grande longueur de cohérence.

Energie. - L'énergie de l'oscillateur a été mesurée au moyen d'une pastille de silicium (étalonnée à $100 \mathrm{mV} / \mathrm{J}$ pour $1,06 \mu$ ) de diamètre d'entrée $2 \mathrm{~mm}$. Nous avons étudié l'énergie monomode en fonction $\mathrm{du}$ niveau de pompage. Pour $10,5 \mathrm{kV}$ nous avons mesuré $20 \mathrm{~mJ}$ à la sortie de l'oscillateur.

Après amplification, l'énergie totale a été mesurée au moyen d'un calorimètre à cône de graphite. Nous avons obtenu $10 \mathrm{~J}$ pour une configuration monomode de la cavité oscillatrice.

Divergence. - A la sortie de l'oscillateur, nous avons mesuré la divergence totale en photographiant deux impacts sur plaque Kodak $1 Z$, en deux endroits différents de la zone lointaine. Par cette méthode, il n'est pas nécessaire de connaître $\gamma$ (voir équation 2). Il suffit de rester dans le domaine de linéarité du détecteur. Nous avons trouvé la divergence totale :

$$
\varepsilon=(5 \pm 1) \times 10^{-4} \mathrm{rd} \text {. }
$$

D'après la référence [8], la divergence théorique d'une onde gaussienne est

$$
\varepsilon_{\mathrm{th}}=\frac{\lambda}{\pi a}=\frac{1,06 \times 10^{-3}}{\pi 0,8}=4 \times 10^{-4} \mathrm{rd} .
$$

Ainsi, aux erreurs d'expérience près, la divergence à la sortie de l'oscillateur est limitée par la diffraction.

Après amplification, nous avons mesuré la divergence en étudiant la distribution d'énergie dans le plan focal d'une lentille (distance focale 1,56 m). Nous avons observé des taches de diffraction (enregistrées sur plaque $1 Z$ ) de diamètre $165 \pm 18 \mu$, ce qui donne la divergence

$$
\varepsilon^{\prime}=\frac{r}{f}=\frac{83 \times 10^{-4}}{156}=(5 \pm 1) \times 10^{-5} \mathrm{rd}
$$

Luminance. - La luminance moyenne est

$$
L=\frac{E}{S} \frac{\tau}{w}
$$

où $E$ est l'énergie totale, $\tau$ la largeur d'impulsion, $S$ la surface émettrice, et $w$ l'angle solide de divergence. D'après l'équation (6), pour une impulsion normale de $40 \mathrm{~ns}$ la luminance à la sortie de l'oscillateur est

$$
L_{\text {osc }}=(9,8 \pm 0,1) \times 10^{13} \mathrm{~W} / \mathrm{St} . \mathrm{cm}^{2} .
$$

Après le second amplificateur, nous avons obtenu la luminance moyenne

$$
L_{\text {tot }}=(1,7 \pm 0,6) \times 10^{16} \mathrm{~W} / \mathrm{St} . \mathrm{cm}^{2} .
$$

\section{Bibliographie}

[1] Hercher (M.), Appl. Phys. Letters, 1965, 7, 39.

[2] Daneu (V.), Sacchi (C. A.) et Svelto (O.), I. E. E. E. J. of Quantum Electronics, 1966, QE 2, 290.

[3] La Macchia (J. T.) et Buorkholm (J. E.), Appl. Phys. Letters, 1968, $12,45$.

[4] Buorkholm (J. E.) et Stolen (R. H.), J. of Appl. Phys., 1968, 39, 4043.

[5] Hirth (A.), Compt. Rend. Acad. Sc., 1969, 268 B, 961.
[6] Hagen (W. F.), J. of Appl. Phys., 1969, 40, 511.

[7] Gerke (P. P.), Denisyuk (Yu.) et LoKshin (V. I.), Soviet J. of Opt. Technology, 1968, 35, 437.

[8] Kogelnik (H.) et Li (T.), Proc. I. E. E. E., 1966, 54, 1312.

[9] Fox (A. G.), et Li (T.), Bell Syst. Techn. J., 1961, 40, 453. 